\title{
Deciphering Modifications in Swine Cardiac Troponin I by Top-Down High-Resolution Tandem Mass Spectrometry
}

\author{
Jiang Zhang, ${ }^{\mathrm{a}, \mathrm{b}}$ Xintong Dong, ${ }^{\mathrm{a}}$ Timothy A. Hacker, ${ }^{\mathrm{c}}$ and Ying $\mathrm{Ge}{ }^{\mathrm{a}, \mathrm{d}}$ \\ ${ }^{a}$ Human Proteomics Program, School of Medicine and Public Health, University of Wisconsin-Madison, \\ Madison, Wisconsin, USA \\ $\mathrm{b}$ The School of Pharmacy, University of Wisconsin-Madison, Madison, Madison, Wisconsin, USA \\ c Department of Medicine, School of Medicine and Public Health, University of Wisconsin-Madison, \\ Madison, Wisconsin, USA \\ d Department of Physiology, School of Medicine and Public Health, University of Wisconsin-Madison, \\ Madison, Wisconsin, USA
}

Cardiac troponin I (cTnI) is an important regulatory protein in cardiac muscle, and its modification represents a key mechanism in the regulation of cardiac muscle contraction and relaxation. cTnI is often referred to as the "gold-standard" serum biomarker for diagnosing patients with acute cardiac injury since it is unique to the heart and released into the circulation following necrotic death of cardiac tissue. The swine (Sus scrofa) heart model is extremely valuable for cardiovascular research since the heart anatomy and coronary artery distribution of swine are almost identical to those of humans. Herein, we report a comprehensive characterization of the modifications in swine cTnI using top-down high-resolution tandem mass spectrometry in conjugation with immunoaffinity chromatography purification. Highresolution high accuracy mass spectrometry revealed that swine cTnI affinity purified from domestic pig hearts was N-terminally acetylated and phosphorylated. Electron capture disassociation is uniquely suited for localization of labile phosphorylations, which unambiguously identified Ser22/Ser23 as the only basally phosphorylated sites that are well-known to be regulated by protein kinase A and protein kinase C. Moreover, a combination of tandem mass spectrometry with sequence homology alignment effectively localized a single amino acid polymorphism, V116A, representing a novel genetic variant of swine cTnI. Overall, our studies demonstrated the unique power of top-down high-resolution tandem mass spectrometry in the characterization of protein modifications, including labile phosphorylation and unexpected sequence variants. (J Am Soc Mass Spectrom 2010, 21, 940-948) @ 2010 American Society for Mass Spectrometry

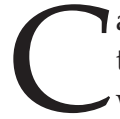
ardiac troponin I (cTnI) is an important regulatory protein in the cardiac muscle. Its interaction with tropomyosin $(\mathrm{Tm})$ and other binding partners within the troponin protein complex represents a key mechanism in the regulation of cardiac muscle contraction and relaxation [1, 2]. cTnI is often referred to as the "gold-standard" serum biomarker for diagnosing patients with acute cardiac injury since it is uniquely present in the heart and released into the general circulation following necrotic death of heart muscle tissue [3]. As the inhibitory subunit of the troponin protein complex, cTnI binds tightly to actin-Tm in the absence of $\mathrm{Ca}^{2+}$ and therefore inhibits the binding of myosin crossbridges to actin. Modification of cTnI changes the conformation of the protein and thereby switches on and off the binding positions on the Tm-

Address reprint requests to Dr. Y. Ge, Human Proteomics Program and Department of Physiology, School of Medicine and Public Health, University of Wisconsin-Madison, 1300 University Ave., SMI 130, Madison 53706, Wisconsin, USA. E-mail: yge@physiology.wisc.edu actin complex to modulate muscle contraction and relaxation. It has been shown that altered modifications of cTnI are directly linked to cardiac dysfunctions in various human heart diseases [4]. Altered phosphorylation of cTnI may contribute causally to cardiac dysfunction in the transition from compensated hypertrophy to heart failure [5, 6]. Proteolytic cleavages of cTnI have been correlated with myocardial stunning and ischemia/reperfusion injury [7]. Mutations in the cTnI gene (TNNI3) have been identified in human familial hypertrophic cardiomyopathies (HCM) [8] and dilated cardiomyopathies (DCM) [9].

The domestic pig (Sus scrofa) is an established mammalian animal model for human diseases [10-14]. Its heart anatomy and coronary artery distribution are almost identical to those of humans [15], making it an extremely valuable animal model for cardiovascular research [13, 14]. Furthermore, the myofilament protein make-up of domestic pig heart shows greater similarity to humans than rodents, implying a highly analogous 
functional role [16]. However, the domestic pig is also known to have very high genetic variability and complexity $[17,18]$, which makes it challenging to use as a research model. A full-characterization of cardiac regulatory proteins such as swine $\mathrm{cTnI}$ and its modifications are essential for understanding its role in cardiac function and thereby establishing proper swine heart models.

The top-down mass spectrometry (MS) methodologies [19-33] have unique advantages in assessing protein modifications, including post-translational modifications (PTMs) and sequence variants. In the conventional bottom-up MS approach, proteins are digested into many small peptides for MS analysis, resulting in partial sequence coverage and loss of connections between disparate portions of proteins containing modifications [34]. Top-down MS approach directly analyzes intact proteins, providing a "bird's eye" view of the protein and reveals all possible modifications present in vivo without a priori knowledge $[35,36]$. Individual protein modifications are then isolated in the gas phase and directly subjected to tandem mass spectrometry (MS/MS) analysis for detailed structural characterization, thus allowing a highly reliable mapping of the modification sites with full sequence coverage $[28,29,37]$. In addition, it greatly simplifies sample preparation and reduces sample complexity as no proteolytic digestion is required. Top-down MS is considerably enhanced with multiple MS/MS fragmentation techniques, including electron capture dissociation (ECD) [38], collisionally activated dissociation (CAD) [39], and infrared multiphoton dissociation (IRMPD) [40] to provide complementary information for comprehensive protein structural characterization. Top-down MS with ECD becomes an extremely valuable tool in mapping labile protein modifications [21, 41], most notably phosphorylation [28-30, 42].

Herein, we have employed an integrated top-down MS strategy combining affinity chromatography for effective purification of cTn from left ventricles of swine myocardium and top-down high-resolution MS/MS for comprehensive analysis of swine cTnI and its modifications. We have thoroughly characterized all the modifications of swine cTnI present in vivo, localized the acetylation and phosphorylation sites, and discovered a novel single amino acid polymorphism that represents a new genetic variant of swine cTnI.

\section{Experimental}

\section{Chemicals and Reagents}

All reagents were purchased from Sigma Chemcial Co. (St. Louis, MO, USA) unless noted otherwise. Complete protease and phosphatase inhibitor cocktail tablets were purchased from Roche Diagnostics Corp. (Indianapolis, IN, USA). All solutions were prepared in Milli-Q water (Millipore Corp., Billerica, MA, USA).

\section{Immunoaffinity Purification of Swine cTn}

Swine heart tissues were obtained from juvenile Yorkshire domestic pig as approved by the University of Wisconsin Animal Care and Use Committee. The excised heart tissue was immediately frozen in liquid nitrogen and stored in a $-80{ }^{\circ} \mathrm{C}$ freezer. The whole cardiac troponin complex was purified by the immunoaffinity purification method as previously described [29]. Briefly, $\sim 1.5 \mathrm{~g}$ of heart tissue was homogenized in tissue wash buffer $\left(\mathrm{NaH}_{2} \mathrm{PO}_{4} 500 \mathrm{mM}, \mathrm{Na}_{2} \mathrm{HPO}_{4} 100\right.$ $\mathrm{mM}, \mathrm{MgCl}_{2} 100 \mathrm{mM}$, EGTA $100 \mathrm{mM}, \mathrm{NaCl} 0.1 \mathrm{M}$, Triton X-100 1\%, DTT $5 \mathrm{mM}$, protease and phosphatase inhibitor cocktail tablet, PMSF $1 \mathrm{mM}$, leupeptin $2 \mu \mathrm{g} / \mathrm{mL}, \mathrm{pH}$ 7.4) using a Polytron electric homogenizer (Model PRO200, PRO Scientific Inc., Oxford, CT, USA) for $30 \mathrm{~s}$ on ice. The homogenate was centrifuged at $8000 \mathrm{rpm}$ for $10 \mathrm{~min}$ at $4{ }^{\circ} \mathrm{C}$; the supernatant was discarded and the pellet was resuspended in $6 \mathrm{~mL}$ protein extraction buffer $(0.7 \mathrm{M} \mathrm{LiCl}, 25 \mathrm{mM}$ Tris, $5 \mathrm{mM}$ EGTA, $0.1 \mathrm{mM}$ $\mathrm{CaCl}_{2}, 5 \mathrm{mM}$ DTT, $1 \mathrm{mM}$ PMSF, $2 \mu \mathrm{g} / \mathrm{mL}$ leupeptin, and $0.75 \mathrm{mg} / \mathrm{mL}$ protease and phosphatase inhibitor cocktail, $\mathrm{pH}$ 8.0) and protein extraction was performed with agitation on a nutating mixer (Fisher Scientific Inc., Pittsburgh, PA, USA) at $4{ }^{\circ} \mathrm{C}$ for $45 \mathrm{~min}$. The sample was then centrifuged at 16,000 rpm (Centrifuge 5415R; Eppendorf, Hamburg, Germany) for 5 min to collect the supernatant. The collected supernatant was further centrifuged at 55,000 rpm (Beckman L-55 ultracentrifuge; Beckman Coulter, Fullerton, CA, USA) for $45 \mathrm{~min}$ to completely remove the tissue debris before affinity chromatography purification. The supernatant was incubated with $0.25 \mathrm{~mL}$ of CNBr-activated Sepharose CL-4B conjugated with $1.25 \mathrm{mg}$ monoclonal cTnI antibody (anti-troponin I monoclonal antibody MF4 and 14G5; HyTest Ltd., Turku, Finland) for $35 \mathrm{~min}$ at $4{ }^{\circ} \mathrm{C}$ to ensure complete binding of troponin complex to the antibody. After washing the column with $2 \mathrm{~mL}$ of extraction buffer, the bound troponin complex was eluted with $100 \mathrm{mM}$ glycine at $\mathrm{pH} 2$ into four $0.4 \mathrm{~mL}$ fractions and neutralized immediately with $40 \mu \mathrm{L}$ of $1 \mathrm{M}$ MOPS (pH 9). Fractions were analyzed for enriched protein content by $15 \%$ sodium dodecyl sulfate polyacrylamide gel electrophoresis (SDS-PAGE) gels stained with Coomassie blue. Typically, $\sim 200 \mu \mathrm{g}$ of $\mathrm{cTnI}$ was recovered from each affinity purification experiment. Analytical repeatability and reproducibility were assessed with three technical replicates (independent purification experiments) for two biological replicates.

\section{Top-Down Mass Spectrometry of Swine cTnI}

Immunoaffinity purified swine cTn complexes were separated and desalted using an offline reverse phase protein microtrap (Michrom Bioresources, Inc., CA, USA), with a two step reverse phase gradient elution method, first with $1 \%$ acetic acid in 30:70 methanol/ water and then $1 \%$ acetic acid in 75:25 methanol/water. Desalted samples were analyzed using a 7T linear 
trap/FTICR (LTQ FT Ultra) hybrid mass spectrometer (Thermo Scientific Inc., Bremen, Germany) equipped with an automated chip-based nano ESI source (Triversa NanoMate; Advion BioSciences, Ithaca, NY, USA). The spray voltage was $1.2-1.6 \mathrm{kV}$ versus the inlet of the mass spectrometer, resulting in a flow of 50-200 nL/ min. Ion transmission into the linear trap and subsequently into the FTICR cell was automatically optimized for maximum ion signal. The number of accumulated ions for the full scan linear trap (IT), FTICR cell (FT), MS ${ }^{n}$ FTICR cell, and ECD were $3 \times 10^{4}$, $8 \times 10^{6}, 8 \times 10^{6}$, and $8 \times 10^{6}$, respectively. The resolving power of the FTICR mass analyzer was typically set at 200,000 at $m / z 400$, resulting in an acquisition rate of 1 scan/s. For CAD and ECD fragmentation, individual charge states of protein molecular ions were first isolated and then dissociated using 10\%-25\% normalized collision energy (CAD) or $2 \%-3 \%$ electron energy (ECD) with a 45-125 ms duration with no delay. Typically, 1000 to 3000 transients were averaged to ensure high quality ECD spectra.

All FTICR spectra were processed with Xtract Software (FT programs 2.0.1.0.6.1.4, Xcalibur 2.0.5, Thermo Scientific Inc., Bremen, Germany) using S/N threshold of 1.5 and fit factor of $40 \%$ and validated manually. The resulting mass lists were further assigned using inhouse developed "Ion Assignment" software (version 1.0) based on the protein sequence of swine cTnI obtained from Swiss-Prot protein knowledgebase (UnitProtKB/Swiss-Prot). Allowance was made for possible PTMs such as the removal of initial Met, acetylation of the N-terminus, and variable phosphorylation sites (residues Ser, Thr, and Tyr), using a 10 and 20 ppm tolerance for precursor and fragment ions, respectively. The assigned ions were manually validated to ensure the quality of assignments. For fragment ions containing possible phosphorylation sites, the mass of fragment ions were manually examined for 80 Da mass shifts to confirm or exclude the existence of phosphorylation. All reported masses are the most abundant masses. The MS signal intensity values were used to calculate the relative ratios for all the observed protein ions based on a method developed by Kelleher and coworkers known as the protein ion relative ratio (PIRR) [43] with modifications [29, 30]. The top five most abundant isotopomer peak heights were integrated to calculate the relative abundance of the intact protein or fragmentation ions.

\section{Results and Discussion}

\section{High-Resolution Mass Spectrometry Analysis of Swine cTnI}

The SDS-PAGE analysis of the immunoaffinity purified proteins revealed three major bands (Supplemental Figure 1, which can be found in the electronic version of this article) representing the three subunits of cTn protein complex, cTnC, cTnI, and cTnT, respectively.
The cTn subunits were further separated and desalted via an offline reverse phase HPLC using a two step gradient elution method and subjected to highresolution FTMS analysis. Figure 1a shows the broadband FTMS spectrum of swine cTnI fraction in 75:25 methanol:water with $1 \%$ acetic acid. High-resolution FTMS analysis of swine cTnI fractions revealed the presence of three major molecular weights $(23,951.76$,

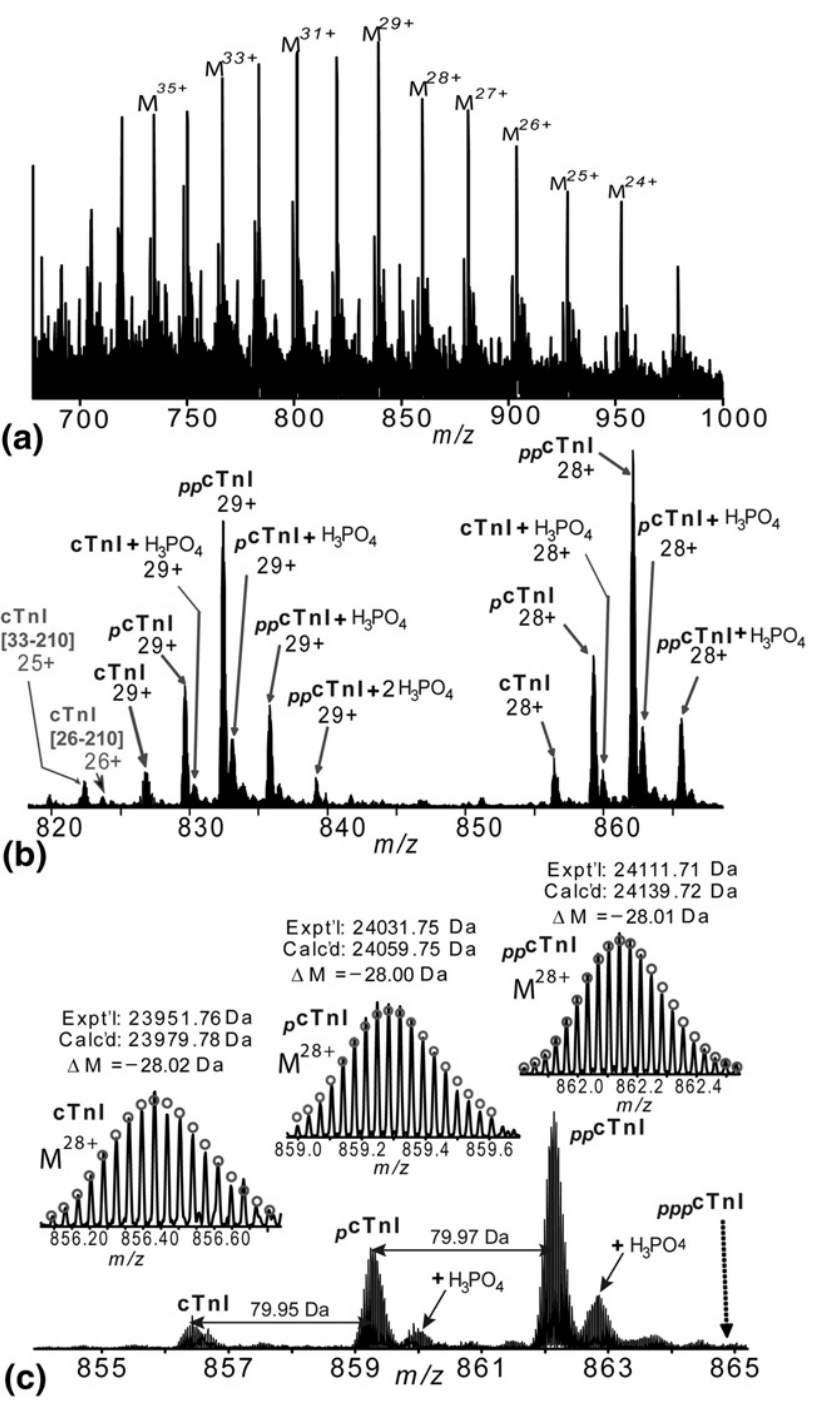

Figure 1. High-resolution MS analysis of intact cTnI purified from domestic pig hearts. (a) Broadband ESI/FTMS spectrum of desalted swine cTnI sample. (b) Expanded MS spectrum of swine cTnI from $m / z 818$ to 868 . (c) Narrow band FTMS spectrum of swine cTnI for the charge state $28^{+}$precursor ions, showing its distribution in un-, mono-, and bisphosphorylated forms. Insets: isotopically resolved molecular ions of un-, mono-, and bisphosphorylated cTnI $\left(\mathrm{M}^{28+}\right)$ with high accuracy molecular weight measurements. ${ }_{p} \mathrm{cTnI}$ and ${ }_{p p} \mathrm{cTnI}$ represent mono- $(+79.95 \mathrm{Da})$ and bisphosphorylated (+159.92 Da) cTnI, respectively. Dashed arrow indicates the expected position for trisphosphorylated cTnI $\left(_{p p p} \mathrm{CTnI}\right)$, which is not observed here. Circles represent the theoretical isotopic abundance distribution of the isotopomer peaks corresponding to the assigned mass. Calc'd: calculated most abundant mass; Expt'l: experimental most abundant mass. $+\mathrm{H}_{3} \mathrm{PO}_{4}$ : noncovalent adduct of phosphoric acid (+98 Da). 


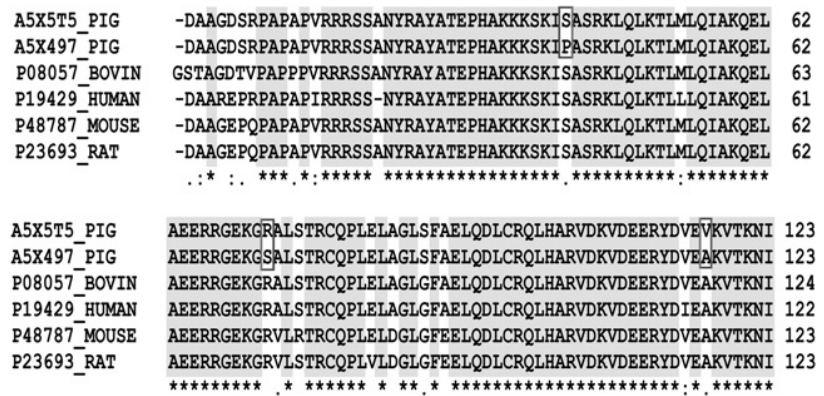

Figure 2. Partial sequence alignment of cTnI from several mammalian species using a Clustal (2.0.11) algorithm. Sequences were downloaded from UnitProtKB/Swiss-Prot. Two splicing isoforms of pig cTnI sequences were aligned with cTnI sequences of bovine, human, mouse, and rat. Numerals indicating amino acid positions are according to the full sequence with the initiating N-terminal Met. Amino acid differences between two swine cTnI isoforms were highlighted in rectangles.

$24,031.75,24,111.71)$, with respective mass increments of 79.96 Da suggesting phosphorylation of swine cTnI (Figure 1b, c). However, none of the observed molecular weights matched with the two widely reported swine cTnI sequences in UniProtKB/SwissProt protein database (http://www.uniprot.org/uniprot/), A5X5T5_PIG and A5X497_PIG from gene TNNI3 (Figure 2, top two sequences, 211 amino acid residues, 24,068.81 Da and $23,982.48 \mathrm{Da}$, respectively), even with consideration of common PTMs such as possible deletion of initiating Met and acetylation. So we applied both ECD and CAD to individually isolated precursor ions of 23,951.76 Da, presumably the unphosphorylated cTnI form, to identify the correct sequence. The detected product ions were assigned with these two reported swine cTnI sequences that differ in three residues S42/P42, R74/ S74, and V116/A116 (Supplemental Figure 2). For both sequences, the product ions gave identical assignments in the N-terminus before S42/P42. After S42, the detected product ions continued to assign larger $c$ and $b$ ions based on the sequence A5X5T5_pig, including $c_{42}$, $c_{45}, c_{47}, c_{48}, c_{49}, c_{50}, c_{51}, c_{53}, c_{54}, c_{55}, c_{58}, c_{59}, c_{60}, c_{63}, c_{65}, c_{67}$, $\boldsymbol{c}_{70}, \boldsymbol{c}_{71}, \boldsymbol{c}_{74}$, and $\boldsymbol{b}_{53}, \boldsymbol{b}_{54}, \boldsymbol{b}_{58}, \boldsymbol{b}_{66}, \boldsymbol{b}_{84}, \boldsymbol{b}_{85}, \boldsymbol{b}_{86}, \boldsymbol{b}_{87}, \boldsymbol{b}_{89}, \boldsymbol{b}_{115}$, while these matches were absent for the sequence A5X497_pig (Supplemental Figure 2). ECD spectrum of 23,951.76 Da matched extensively with A5X5T5_PIG, after deletion of N-terminal Met and incorporation of acetylation at the new N-terminus (Supplemental Figure 3). Overall, the MS/MS data suggested that sequence of A5X5T5_PIG is the better match for the swine cTnI analyzed here rather than A5X497_pig. The other two molecular masses, 24,031.75 Da and 24,111.71 Da, were assigned as monophosphorylated and bisphosphorylated cTnI, respectively, based on highly accurate molecular mass measurements (Figure 1c insets). Nevertheless, the high accuracy mass measurements of the three major cTnI forms (Figure 1c) clearly showed a mass discrepancy $(-28 \mathrm{Da})$ between the experimental and calculated masses of swine cTnI (A5X5T5_PIG) with consideration of acetylation and phosphorylation.
As shown in Figure 1b, only minor degradation products of cTnI assigned as P[33-210]G and Y[26-210]G were observed in this sample, indicating the heart samples were well-preserved during the storage and purification procedures.

\section{Localization of Phosphorylation Sites in Swine cTnI}

The relative intensity ratio for cTnI in its unphosphorylated (cTnI), monophosphorylated $\left({ }_{p} \mathrm{cTnI}\right)$, and bisphosphorylated ${ }_{p p} \mathrm{cTnI}$ ) forms was $\sim 6.8 \% \pm 1.3 \%$ $27.8 \% \pm 1.4 \%$, and $64 \% \pm 2.7 \%$, respectively, indicating that swine cTnI was predominantly basally phosphorylated. No trisphosphorylated cTnI was detected, implying higher order phosphorylation was not present in the swine cTnI sample analyzed, or below the detection limit of the FTMS method used in this study (estimated to be $<1 \%$ of the total cTnI protein population based on the signal intensity at the expected position of trisphosphorylated cTnI over the summed intensity of all cTnI forms).

To determine the phosphorylation sites, the precursor ions of bisphosphorylated cTnI were isolated and fragmented by both ECD and CAD. One ECD spectrum generated 175 validated high quality MS/MS peaks (Figure 3). No phosphorylated product ions were detected for $c_{19}, c_{20}$, and $c_{21}$, as well as other smaller $c$ ions, which provided unambiguous evidence that the first 21

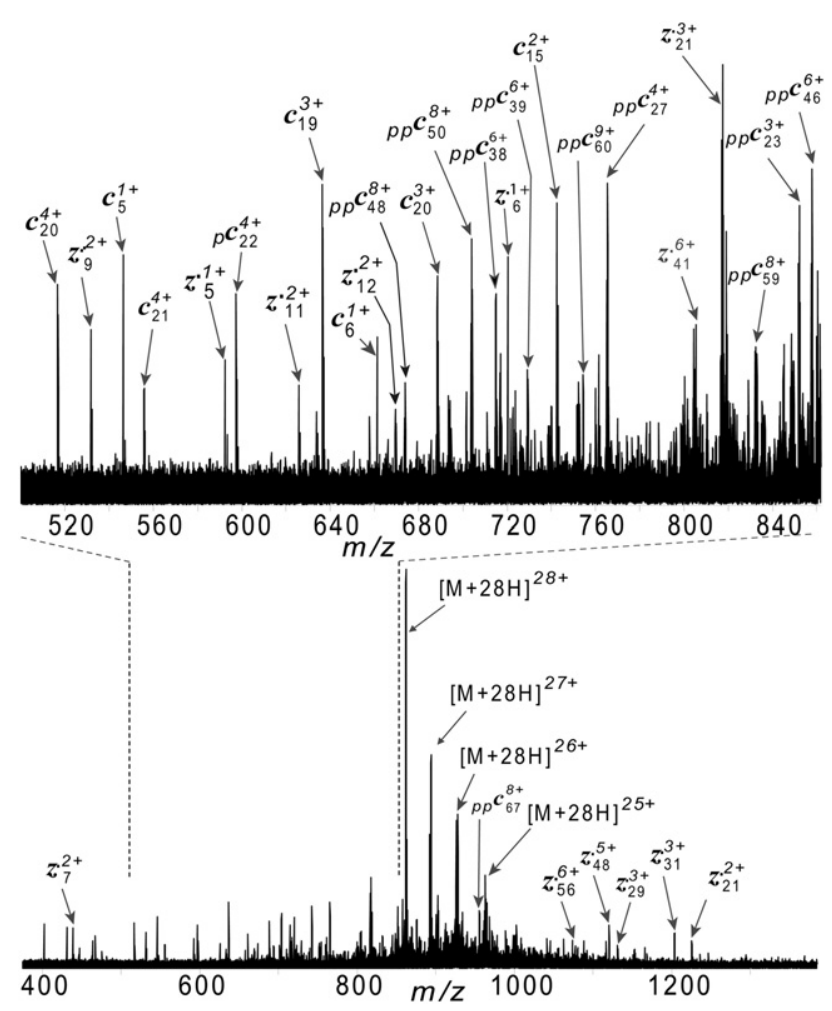

Figure 3. ECD spectrum of isolated bisphosphorylated cTnI $\left({ }_{p p} \mathrm{CTnI}\right)$. The product ion assignments were made according to the sequence A5X5T5_PIG with the removal of N-terminal Met and acetylation at the new terminus. 
amino acid residues do not contain the phosphorylation sites (Figure $4 \mathrm{a}, \mathrm{b}, \mathrm{c}$ ). In contrast, monophosphorylated $c_{22}$ ions $\left({ }_{p} c_{22}\right)$ was detected, which clearly indicated the phosphorylation of Ser22 (Figure $4 \mathrm{~d}$ ). No unphosphorylated $c_{22}$ was detected (estimated to be $<4 \%$ of the monophosphorylated $c_{22}$ ions), which suggested Ser22 was completely phosphorylated and confirmed there was no phosphoryl group loss during ECD fragmentation. Similarly, bisphosphorylated $c_{23}$ ions $\left({ }_{p p} c_{23}\right)$ were also detected, indicating the successive phosphorylation on Ser23 (Figure 4e). No unphosphorylated or monophosphorylated counter ions were detected for ${ }_{p p} \mathrm{C}_{23}$ and ${ }_{p p} \mathrm{C}_{27}$ (estimated to be $<2 \%-3 \%$ of the bisphosphorylated $c_{23}$ and $c_{27}$ ions), which revealed the full phosphorylation occupancy on Ser22 and Ser23 (Figure $4 \mathrm{e}, \mathrm{f})$. Conversely, if other phosphorylation sites exist in the bisphosphorylated cTnI, one would expect to ob-

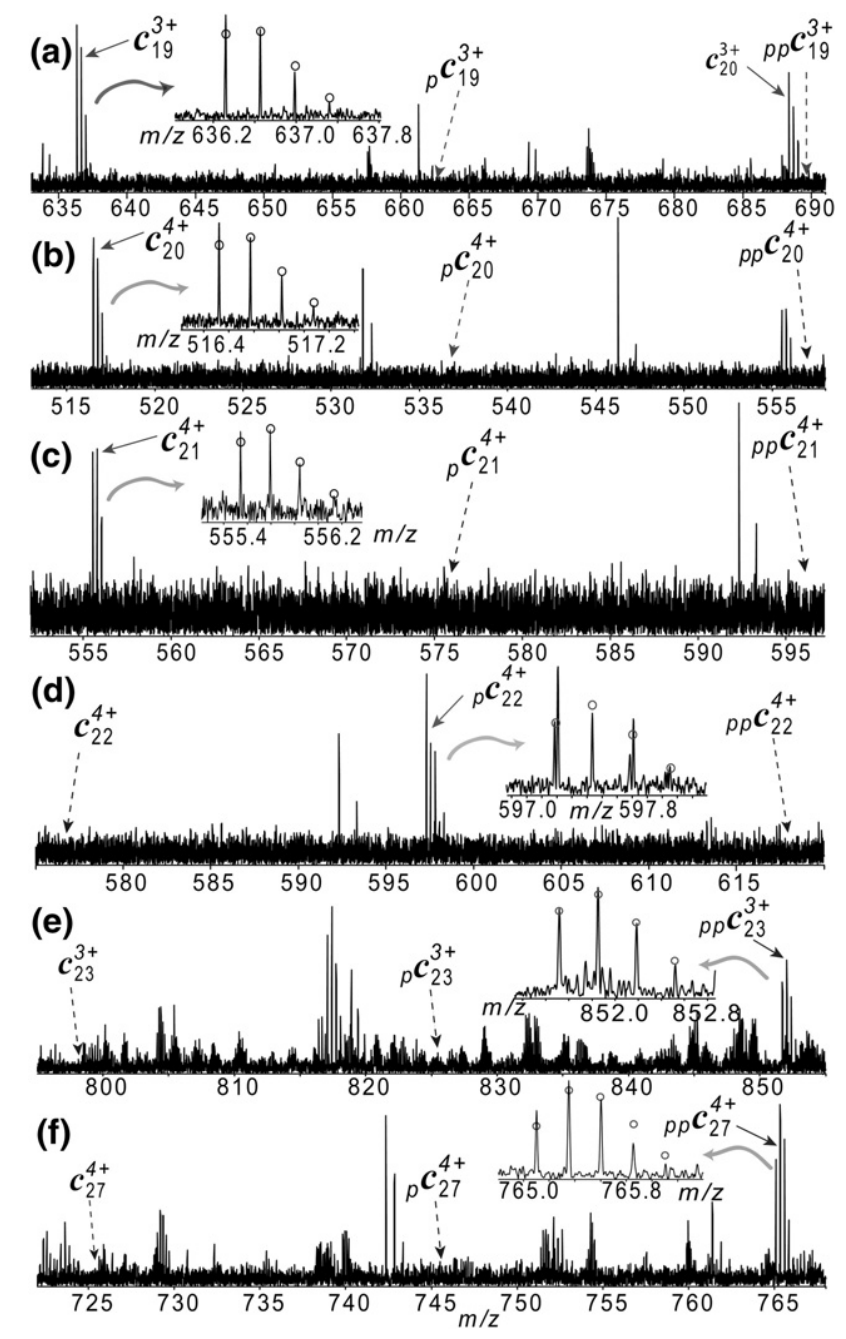

Figure 4. Key product ions (a-f) from the ECD spectra for localization of swine cTnI bisphosphorylation sites. Solid arrows indicate the observed product ions; dashed arrows indicate the absence of the labeled product ions. Circles represent the theoretical isotopic abundance distribution of the isotopomer peaks corresponding to the assigned mass. ${ }_{p} c$ and ${ }_{p p} c$ stand for monoand bisphosphorylated $c$ ions. serve un- and monophosphorylated $c_{22}, c_{23}$, and $c_{27}$ ions, which were not observed in our experiments.

The identification of Ser22/23 as the only phosphorylation sites is consistent with our previous report on a mouse cTnI [29]. Phosphorylation of Ser22/23 are wellknown to be regulated by protein kinase A (PKA) upon $\beta$-adrenergic stimulation [44]. Protein kinase C (PKC, $\delta / \beta / \varepsilon$ isozymes) can cross-phosphorylate the PKA sites of cTnI at Ser22/23 as well as protein kinase D (PKD) and cyclic GMP-dependent protein kinase (PKG) [4547] [6]. Our previously reported human cTnI study also revealed that Ser22/23 are the two basally phosphorylated sites [28]. The cTnI sequences of these closely related mammalian species also showed striking similarities (Figure 2), with a 93\% sequence homology between mouse, pig, and human. The sequence similarity is even higher between close members within the same family, such as mouse and rat (99\% sequence homology). The conservation of cTnI basal phosphorylation sites within multiple mammalian species implies a highly analogous functional role of $\mathrm{cTnI}$ in regulating cardiac contraction and relaxation.

The phosphorylation of cTnI has been well-studied and demonstrated to be essential for modulating cardiac function [2, 4-6]. Apart from the Ser22/23 (known as Ser23/24 considering the N-terminal Met) phosphorylation sites as identified here, there is a significant body of literature demonstrating the phosphorylation sites at Ser42/44 and Thr143 (Ser43/45 and Thr144 with the N-terminal Met) [2, 6, 45-49]. However, these other phosphorylation sites (Ser42/44 and Thr143) were identified from in vitro phosphorylation assays. In contrast, the sites identified in this study (Ser22/23) are basally presented in swine cTnI in vivo. Ser22/23 are also the only sites basally phosphorylated in wild-type healthy mice, which has been systematically investigated in our previous publication [29]. The observation of basal phosphorylation sites at Ser22/23 does not lessen the significance of the PKC phosphorylation as discussed previously [29]. Ser42/44 and Thr143 are the substrates for protein kinase C (PKC) [2, 6, 45-49] and PKC was shown to be up-regulated in the end stage heart failure [50]. Thus, it is reasonable to suspect that these PKC sites may be considered maladaptive and connected with disorders of the heart [2]. A heart at the end stage failure is substantially different from a healthy one at the molecular and cellular level [49]. Please note that the cTnI analyzed here is from healthy pig hearts, so their phosphorylation state could be different from those of diseased hearts. Another possibility is that the PKC sites may present in extremely low phosphorylation occupancy in cTnI that are too low to be detected. Since every analytical method has its own detection limit, we cannot exclude the possibility of other sites of very low $(<1 \%)$ phosphorylation occupancy.

To address the concern whether affinity purification may selectively enrich certain types of phosphorylated species, we have used antibodies of different epitope regions (N-terminal residues $1-23$ versus $\mathrm{C}$-terminal 
residues 190-197 of cTnI). Our results consistently showed the same cTnI species were detected regardless of the antibodies used (data not shown). Hence, this suggests that the overall impact of affinity chromatography on the phosphoprotein selection was minimal.

\section{Localization of Single Amino Acid Polymorphism in Swine cTnI}

To locate the mass discrepancy of $-28 \mathrm{Da}$, we applied ECD and CAD directly to the individually isolated unand bisphosphorylated cTnI molecular ions, respectively (Figure 5). Both ECD and CAD fragmentation generated extensive product ions, which matched the $\mathrm{N}$ - and C-terminal ions predicted from the sequence A5X5T5_pig precisely, indicating the mass discrepancy site was not near either $\mathrm{C}$ - or N-termini. The largest fragmentation ions detected in ECD spectra of unphosphorylated (Figure 5a) and bisphosphorylated cTnI (Figure $5 \mathrm{~b}$ ), $c_{74}$ and $z^{\bullet}{ }_{76}, c_{85}$, and $z^{\bullet}{ }_{77}$, respectively, were matched with the sequence A5X5T5_pig with high accuracy. Meanwhile the larger ions observed in ECD of unphosphorylated cTnI (Figure 5a) including $z^{\bullet}{ }_{103}$, $z^{\bullet}{ }_{119}, \boldsymbol{z}^{\bullet}{ }_{172}, \boldsymbol{z}^{\bullet}{ }_{185}, \boldsymbol{z}^{\bullet}{ }_{209}$, and $\boldsymbol{c}_{209}$ all carry a -28 Da mass discrepancy, which restricts such a mass discrepancy to the middle of the sequence D[108-133]F. CAD with mild fragmentation conditions $(10 \%-15 \%$ normalized collisional energy) of the isolated bisphosphorylated cTnI $\left({ }_{p p} \mathrm{cTnI}\right)$ precursor ions produced many larger product ions (Figure 6) including $18 \boldsymbol{b} / y$ complementary pairs with full sequence coverage (Figure $5 b$ ). No $-28 \mathrm{Da}$ mass discrepancies were observed in smaller $\boldsymbol{b} / \boldsymbol{y}$ fragmentation ion series, $\boldsymbol{b}_{3}-\boldsymbol{b}_{115}$ and $\boldsymbol{y}_{4}-\boldsymbol{y}_{88}$. In contrast, all the larger $\boldsymbol{b} / \boldsymbol{y}$ ions, $\boldsymbol{b}_{122}-\boldsymbol{b}_{198}$, and $\boldsymbol{y}_{95}-\boldsymbol{y}_{209}$ contain such a -28 Da mass discrepancy. Hence, the mass discrepancy site was localized to the seven amino acids between V116 and I122,VKVTKNI (Figure 5). Taken together, two ECD and three CAD experiments of the unphosphorylated cTnI generated a total of $40 c$ and $39 z^{\bullet}$ ions and $41 \boldsymbol{b}$ ions and $57 \boldsymbol{y}$ ions, which unambiguously localized the acetylation site to the N-terminus. The overall fragmentation map of the bisphosphorylated cTnI from two ECD and three CAD experiments generated a total of $35 c$ and $41 z^{\bullet}$ ions corresponding to 76 inter-residue bond cleavages, and $43 \boldsymbol{b}$ ions and $51 \boldsymbol{y}$ ions representing 77 bond cleavages with full sequence

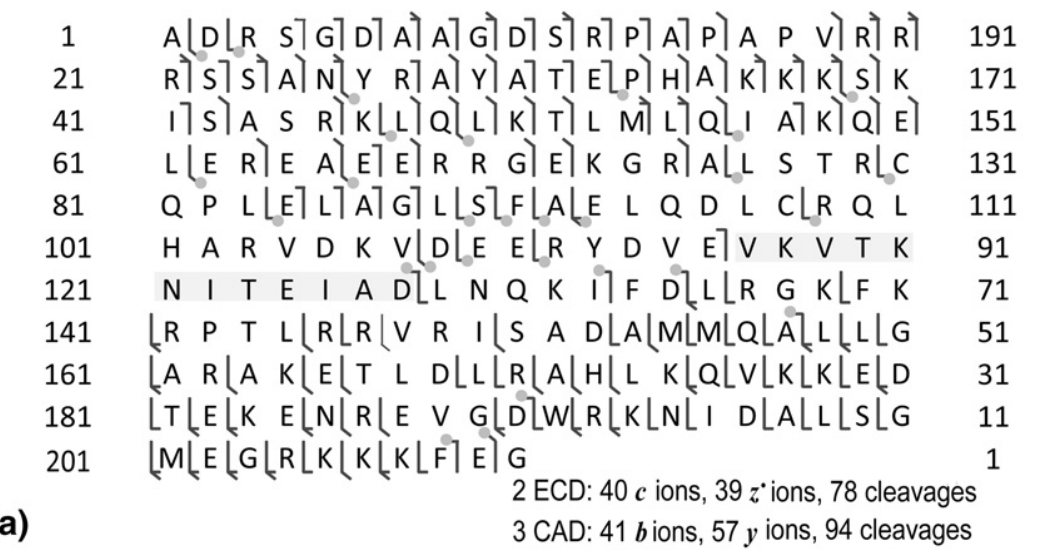

(a) 3 CAD: $41 b$ ions, $57 y$ ions, 94 cleavages

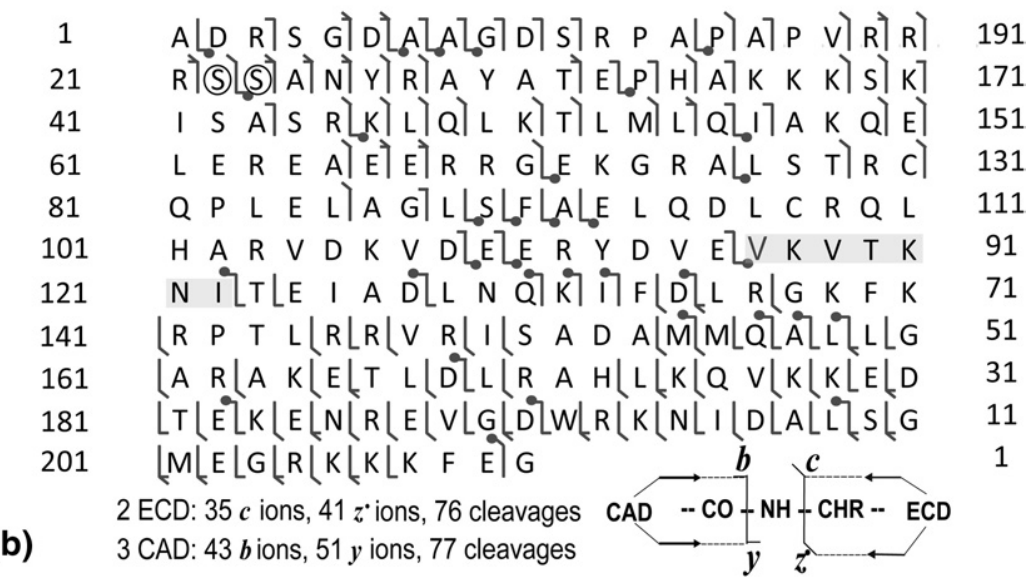

Figure 5. MS/MS fragmentation and product ion map from ECD and CAD spectra for (a) un- and (b) bisphosphorylated swine $\mathrm{cTnI}\left({ }_{p p} \mathrm{cTnI}\right)$. Fragments assignments were made to the swine cTnI (UnitProtKB/Swiss-Prot A5X5T5, TNNI3_pig) with the removal of N-terminal Met and acetylation at the new terminus. Bisphosphorylation sites of Ser22/23 were highlighted by circles. The fragmentation ions carrying the mass discrepancy $(-28 \mathrm{Da})$ is indicated in dots. The potential amino acids containing the mass discrepancy $(-28 \mathrm{Da})$ are highlighted in shades. 


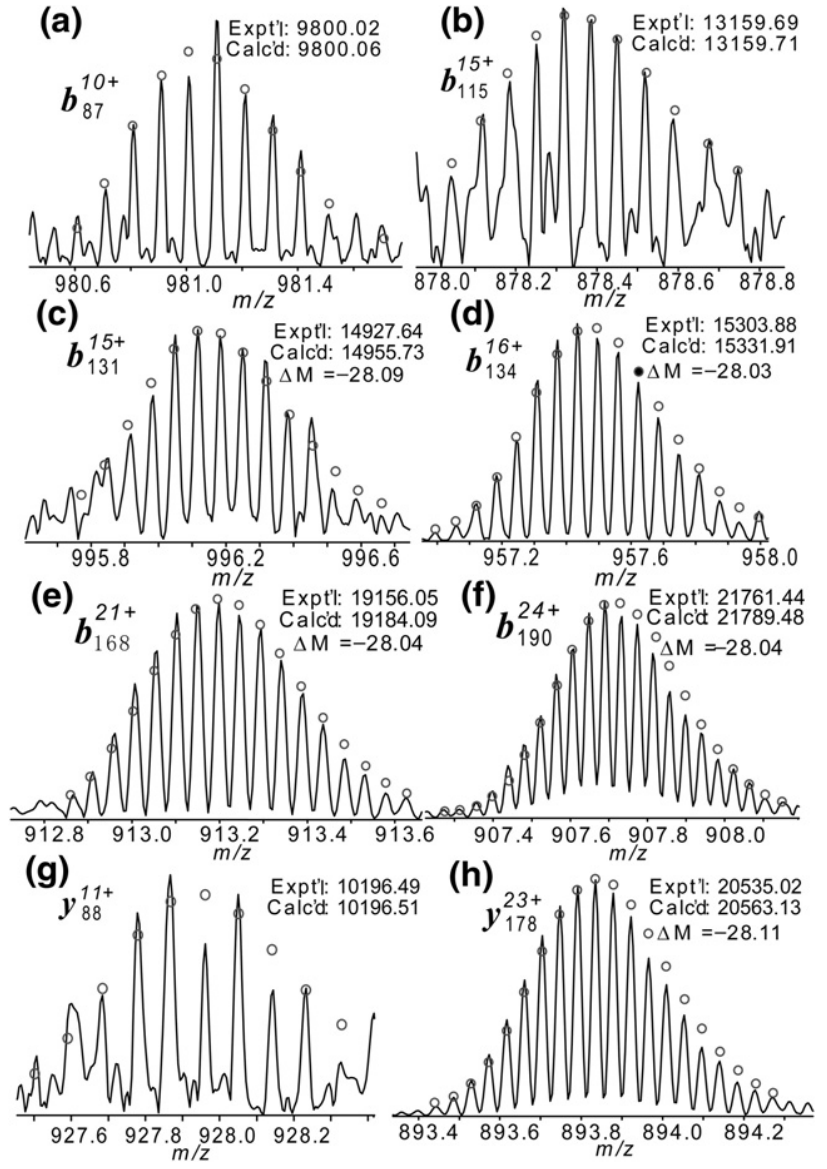

Figure 6. Representative product ions $(\mathbf{a}-\mathbf{h})$ from the CAD spectrum of bisphosphorylated swine $\mathrm{CTnI}$ for localization of the mass discrepancy $(-28 \mathrm{Da})$. Product ions are assigned according to the sequence A5X5T5_pig with the removal of N-terminal Met, acetylation at the new terminus, and phosphorylations at Ser22 and Ser23. Circles represent the theoretical isotopic abundance distribution of the isotopomer peaks corresponding to the assigned mass. Calc'd: calculated most abundant mass; Expt'l: experimental most abundant mass.

coverage. All assigned sequence ions were detected with high mass accuracy with root mean square (RMS) values of 4.6 and $6.3 \mathrm{ppm}$ for ECD and CAD data, respectively.

We performed three independent purification experiments for the two biological replicates, which consistently showed the -28 Da mass discrepancy in swine cTnI (data not shown). The -28 Da mass discrepancy most likely resulted from an amino acid polymorphism rather than a PTM since there is no common protein PTM that corresponds to a -28 Da shift. It is highly unlikely to be a combination of two or more PTMs within such a short amino acid sequence range. A -28 Da mass shift could potentially be generated from amino acid polymorphisms of V/A, D/S, E/T, M/C, and $\mathrm{R} / \mathrm{K}$ (Table 1 ). So the only possibility to account for $-28 \mathrm{Da}$ shift in this sequence region, VKVTKNI, is either V116A or V118A. Moreover, the sequence alignment of cTnI from multiple mammalian species shows that within the seven amino acids region V116-I122, all other amino acids are conserved except V116 (Figure 2). Val is only present in swine cTnI (A5X5T5_Pig) whereas Ala is commonly predicted for amino acid position 116 (117 if including the N-terminal Met) of bovine, human, mouse, and rat cTnI sequences as well as the other sequence variant of swine cTnI (AA5X497_Pig). Therefore, the single amino acid polymorphism in swine cTnI is most likely at amino acid position 116 (V116A). The domestic pig is a species known to be of high genetic variability $[17,18]$. However, currently there are only two reported cTnI sequence variants in the Swiss-Prot and NCBI database from one common ancestor gene TNNI3, with three amino acid polymorphism sites in a total of 211 residues. Hence, the observed cTnI in our experiments represents a novel sequence variant that is first reported here.

\section{Top-Down MS/MS for Determination of Protein Modifications}

As demonstrated here, top-down MS methodology offers unique advantages in assessing protein modifications including PTMs and sequence variants. The MS acquired at the intact protein level (the "top" part) provides a "bird's eye" view of all potential modifications [26, 35] and the MS/MS of the isolated ions of interests (the "down" part) reliably localizes the modification sites. In this study, we have unambiguously identified two basal phosphorylation sites and one novel single amino acid polymorphism of swine cTnI. We found that the two MS/MS techniques, ECD and CAD, are complementary for characterization of PTMs and amino acid polymorphisms. ECD is uniquely suitable for determination of labile modifications such as phosphorylation since it preserves labile modifications during MS/MS process as demonstrated in this study. Such labile modifications have often been "knockedoff" in conventional energetic fragmentation process such as CAD, IRMPD, and post-source decay (PSD). On the other hand, the efficiency of CAD $(70 \%-80 \%)$ is higher than ECD $(10 \%-30 \%)$, which generates more ions for the reliable identification of stable PTMs sites and amino acid polymorphisms. Furthermore, it is more difficult to obtain large fragmentation ions in an ECD spectrum than CAD. Here we have selected a mild energy condition for CAD experiments, which gener-

Table 1. Possible amino acid polymorphisms with $-28 \mathrm{Da}$ mass difference

\begin{tabular}{ccc}
\hline & \multicolumn{2}{c}{ Mass difference (Da) } \\
\cline { 2 - 3 } Polymorphism* $^{*}$ & Monoisotopic & Average \\
\hline \hline V/A & -28.03 & -27.99 \\
D/S & -28.00 & -28.02 \\
E/T & -27.99 & -28.00 \\
M/C & -28.03 & -28.10 \\
R/K & -28.01 & -28.00 \\
\hline
\end{tabular}

${ }^{*} \mathrm{X} / \mathrm{Y}$ stands for the polymorphism of amino acid residue $\mathrm{X}$ to $\mathrm{Y}$. 
ated larger fragmentation ions for effective localization of a single amino acid polymorphism site that is located in the middle of the protein sequence.

Top-down MS methodology is superior in resolving unexpected modifications without a priori knowledge. Given the highly accurate mass measurement and extensive product ion matches from both ECD and CAD, our data revealed a single amino acid polymorphism, suggesting a novel cTnI sequence variant in the domestic pig. Although bottom-up MS appears to be highly efficient for sequencing small tryptic peptides and valuable for large scale high throughput protein identification, it can easily overlook unexpected PTMs or sequence variants. In the bottom-up approach, proteins are digested into smaller tryptic peptides and the MS analysis is performed at the peptide level. The information regarding the whole protein is then pieced together from its peptide components. Such a bottom-up approach relies heavily on the presence of the existing protein sequences in the database and a priori knowledge of expected modifications for identification of protein sequences and the modification sites. Moreover, due to the partial sequence coverage, the bottom-up strategy has a very limited ability to discern proteins with high sequence similarities, let alone single amino acid polymorphisms as identified in this study using a top-down MS approach. In addition, piecing very small tryptic peptides together often results in the loss of connections between disparate portions of proteins containing modifications, thereby limiting the ability for detailed protein structural characterization. On the contrary, in the top-down MS approach, all PTMs and sequence variations can be resolved at the molecular ion level without a priori knowledge [26]. MS/MS fragmentation is directly performed at the intact protein level, ensuring full protein sequence coverage for reliable mapping of PTMs [37] and amino acid polymorphisms (also referred to as "protein-sequence polymorphisms") [27, 51]. The ionization efficiency of intact proteins is much less affected by the presence of a modifying group in comparison to peptides, thus making top-down MS especially attractive for quantifying modified positional isomers [28, 43] and exploring interdependency among multiple modification sites [29, 30].

\section{Conclusions}

An integrated top-down protein MS strategy combining immuno-affinity chromatography and high-resolution FTMS/MS was employed here to study the modification state of swine cTnI purified from domestic pig heart tissues. We have comprehensively characterized all the modifications in swine cTnI, including acetylation, phosphorylation, and sequence variants. The highresolution MS and MS/MS data from the top-down experiments provide solid and unbiased evidence regarding the possible modification sites in the protein sequence as exemplified in this study. Top-down ECD unambiguously identified two basal phosphorylation sites in swine cTnI, which are well conserved among the mammalian species. A combination of ECD and CAD with sequence homology alignment effectively located an unexpected single amino acid polymorphism representing a novel sequence variant of swine cTnI. Taken together, our study illustrated the unique power of the top-down MS strategy in the analysis of protein PTMs and sequence variants in endogenous protein purified directly from tissues. Such an unexpected sequence variant with a single amino acid polymorphism would be extremely challenging and could have been easily overlooked using a bottom-up MS approach.

\section{Acknowledgments}

The authors gratefully acknowledge Professor Neil L. Kelleher for his pioneering contributions to the field of top-down mass spectrometry-based proteomics and congratulate him on his selection as a Biemann awardee. The authors thank Lisa $\mathrm{Xu}$, Huseyin Guner, and Matthew Lawrence in the Human Proteomics Program at UW-Madison for help with instrumentations, and Drs. Wei Xu and Lingjun Li for helpful discussions. Financial support was kindly provided by the Wisconsin Partnership Fund for a Healthy Future and American Heart Association Scientist Development grant 0735443Z (to Y.G.).

\section{Appendix A Supplementary Material}

Supplementary material associated with this article may be found in the online version at doi:10.1016/ j.jasms.2010.02.005.

\section{References}

1. Takeda, S.; Yamashita, A.; Maeda, K.; Maeda, Y. Structure of the Core Domain of Human Cardiac Troponin in the $\mathrm{Ca}^{2+}$-Saturated Form. Nature 2003, 424, 35-41.

2. Solaro, R. J.; Rosevear, P.; Kobayashi, T. The Unique Functions of Cardiac Troponin I in the Control of Cardiac Muscle Contraction and Relaxation. Biochem. Biophys. Res. Commun. 2008, 369, 82-87.

3. Babuin, L.; Jaffe, A. S. Troponin: The Biomarker of Choice for the Detection of Cardiac Injury. Can. Med. Assoc. J. 2005, 173, 1191-1202.

4. Hamdani, N.; Kooij, V.; van Dijk, S.; Merkus, D.; Paulus, W. J.; dos Remedios, C.; Duncker, D. J.; Stienen, G. J. M.; van der Velden, J. Sarcomeric Dysfunction in Heart Failure. Cardiovas. Res. 2008, 77, 649-658.

5. van der Velden, J.; Papp, Z.; Zaremba, R.; Boontje, N. M.; de Jong, J. W.; Owen, V. J.; Burton, P. B. J.; Goldmann, P.; Jaquet, K.; Stienen, G. J. M. Increased $\mathrm{Ca}^{2+}$-Sensitivity of the Contractile Apparatus in End-Stage Human Heart Failure Results from Altered Phosphorylation of Contractile Proteins. Cardiovasc. Res. 2003, 57, 37-47.

6. Layland, J.; Solaro, R. J.; Shah, A. M. Regulation of Cardiac Contractile Function by Troponin I Phosphorylation. Cardiovasc. Res. 2005, 66, 12-21.

7. Murphy, A. M.; Kogler, H.; Georgakopoulos, D.; McDonough, J. L.; Kass, D. A.; Van Eyk, J. E.; Marban, E. Transgenic Mouse Model of Stunned Myocardium. Science 2000, 287, 488-491.

8. Morita, H.; Seidman, J.; Seidman, C. E. Genetic Causes of Human Heart Failure. J. Clin. Invest. 2005, 115, 518-526.

9. Murphy, R. T.; Mogensen, J.; Shaw, A.; Kubo, T.; Hughes, S.; McKenna, W. J. Novel Mutation in Cardiac Troponin I in Recessive Idiopathic Dilated Cardiomyopathy. Lancet 2004, 363, 371-372.

10. Ekser, B.; Rigotti, P.; Gridelli, B.; Cooper, D. K. C. Xenotransplantation of Solid Organs in the Pig-to-Primate Model. Transplant. Immunol. 2009, 21, 87-92.

11. Chinchoy, E.; Soule, C. L.; Houlton, A. J.; Gallagher, W. J.; Hjelle, M. A.; Laske, T. G.; Morissette, J.; Iaizzo, P. A. Isolated Four-Chamber Working Swine Heart Model. Ann. Thorac. Surg. 2000, 70, 1607-1614. 
12. Gross, D. R. Thromboembolic Phenomena and the Use of the Pig as an Appropriate Animal Model for Research on Cardiovascular Devices. Int. J. Artif. Organs 1997, 20, 195-203.

13. Hasenfuss, G. Animal Models of Human Cardiovascular Disease, Heart Failure, and Hypertrophy. Cardiovasc. Res. 1998, 39, 60-76.

14. Crick, S. J.; Sheppard, M. N.; Ho, S. Y.; Gebstein, L.; Anderson, R. H. Anatomy of the Pig Heart: Comparisons with Normal Human Cardiac Structure. J. Anat. 1998, 193, 105-119.

15. Weaver, M. E.; Pantely, G. A.; Bristow, J. D.; Ladley, H. D. A Quantitative Study of The Anatomy And Distribution Of Coronary Arteries in Swine in Comparison with Other Animals and Man. Cardiovasc. Res. 1986, 20, 907-917.

16. Hamdani, N.; de Waard, M.; Messer, A. E.; Boontje, N. M.; Kooij, V.; van Dijk, S.; Versteilen, A.; Lamberts, R.; Merkus, D.; dos Remedios, C.; Duncker, D. J.; Borbely, A.; Papp, Z.; Paulus, W.; Stienen, G. J. M.; Marston, S. B.; van der Velden, J. Myofilament Dysfunction in Cardiac Disease from Mice to Men. J. Muscle Res. Cell Motil. 2008, 29, 189-201.

17. Lunney, J. K. Advances in Swine Biomedical Model Genomics. Int. J. Biol. Sci. 2007, 3, 179-184.

18. Kitamura, S.; Muroya, S.; Nakajima, I.; Chikuni, K.; Nishimura, T. Amino Acid Sequences of Porcine Fast and Slow Troponin T Isoforms. Biosci. Biotechnol. Biochem. 2006, 70, 726-728.

19. Kelleher, N. L.; Lin, H. Y.; Valaskovic, G. A.; Aaserud, D. J.; Fridriksson, E. K.; McLafferty, F. W. Top Down Versus Bottom Up Protein Characterization by Tandem High-resolution Mass Spectrometry. J. Am. Chem. Soc. 1999, 121, 806-812.

20. Kelleher, N. L. Top-down Proteomics. Anal. Chem. 2004, 76, 196A-203A.

21. Kelleher, R. L.; Zubarev, R. A.; Bush, K.; Furie, B.; Furie, B. C.; McLafferty, F. W.; Walsh, C. T. Localization of Labile Posttranslational Modifications by Electron Capture Dissociation: The Case of $\boldsymbol{\gamma}$ Carboxyglutamic Acid. Anal. Chem. 1999, 71, 4250-4253.

22. Ge, Y.; Lawhorn, B. G.; El Naggar, M.; Strauss, E.; Park, J. H.; Begley, T. P.; McLafferty, F. W. Top Down Characterization of Larger Proteins (45 kDa) by Electron Capture Dissociation Mass Spectrometry. J. Am. Chem. Soc. 2002, 124, 672-678.

23. Ge, Y.; El Naggar, M.; Sze, S. K.; Bin Oh, H.; Begley, T. P.; McLafferty, F. W.; Boshoff, H.; Barry, C. E. Top Down Characterization of Secreted Proteins from Mycobacterium tuberculosis by Electron Capture Dissociation Mass Spectrometry. J. Am. Soc. Mass Spectrom. 2003, 14, 253-261.

24. Meng, F. Y.; Forbes, A. J.; Miller, L. M.; Kelleher, N. L. Detection and Localization of Protein Modifications by High-Resolution Tandem Mass Spectrometry. Mass Spectrom. Rev. 2005, 24, 126-134.

25. Han, X. M.; Jin, M.; Breuker, K.; McLafferty, F. W. Extending Top-Down Mass Spectrometry to Proteins with Masses Greater Than 200 Kilodaltons. Science 2006, 314, 109-112.

26. Siuti, N.; Kelleher, N. L. Decoding Protein Modifications Using TopDown Mass Spectrometry. Nat. Methods 2007, 4, 817-821.

27. Sancho Solis, R.; Ge, Y.; Walker, J. W. Single Amino Acid Sequence Polymorphisms in Rat Cardiac Troponin Revealed by Top-Down Tandem Mass Spectrometry. J. Muscle Res. Cell Motil. 2008, 29, 203-212.

28. Zabrouskov, V.; Ge, Y.; Schwartz, J.; Walker, J. W. Unraveling Molecular Complexity of Phosphorylated Human Cardiac Troponin I by Top Down Electron Capture Dissociation/Electron Transfer Dissociation Mass Spectrometry. Mol. Cell. Proteom. 2008, 7, 1838-1849.

29. Ayaz-Guner, S.; Zhang, J.; Li, L.; Walker, J. W.; Ge, Y. In Vivo Phosphorylation Site Mapping in Mouse Cardiac Troponin I by HighResolution Top-Down Electron Capture Dissociation Mass Spectrometry: Ser22/23 Are the Only Sites Basally Phosphorylated. Biochemistry 2009, 48, 8161-8170

30. Ge, Y.; Rybakova, I.; Xu, Q.; Moss, R. L. Top-Down High Resolution Mass Spectrometry of Cardiac Myosin Binding Protein C Revealed That Truncation Alters Protein Phosphorylation State. Proc. Natl. Acad. Sci. U.S.A. 2009, 106, 12658-12663.

31. Whitelegge, J.; Halgand, F.; Souda, P.; Zabrouskov, V. Top-Down Mass Spectrometry of Integral Membrane Proteins. Expert Rev. Proteom. 2006, 3, 585-596.

32. Xie, Y. M.; Zhang, J.; Yin, S.; Loo, J. A. Top-Down ESI-ECD-FT-ICR Mass Spectrometry Localizes Noncovalent Protein-Ligand Binding Sites. J. Am. Chem. Soc. 2006, 128, 14432-14433.
33. Jebanathirajah, J. A.; Pittman, J. L.; Thomson, B. A.; Budnik, B. A.; Kaur, P.: Rape, M.; Kirschner, M.; Costello, C. E.: O'Connor, P. B. Characterization of a New qQq-FTICR Mass Spectrometer for Post-Translational Modification Analysis and Top-Down Tandem Mass Spectrometry of Whole Proteins. J. Am. Soc. Mass Spectrom. 2005, 16, 1985-1999.

34. Chait, B. T. Mass Spectrometry: Bottom-Up or Top-Down? Science 2006 $314,65-66$

35. Thomas, C. E.; Kelleher, N. L.; Mizzen, C. A. Mass Spectrometric Characterization of Human Histone H3: A Bird's Eye View. J. Proteome Res. 2006, 5, 240-247.

36. Jiang, L.; Smith, J. N.; Anderson, S. L.; Ma, P.; Mizzen, C. A.; Kelleher N. L. Global Assessment of Combinatorial Post-Translational Modification of Core Histones in Yeast Using Contemporary Mass Spectrometry. J. Biol. Chem. 2007, 282, 27923-27934

37. Kuhn, P.; Xu, Q. G.; Cline, E.; Zhang, D.; Ge, Y.; Xu, W. Delineating Anopheles gambiae Coactivator Associated Arginine Methyltransferase 1 Automethylation Using Top-Down High-Resolution Tandem Mass Spectrometry. Protein Sci. 2009, 18, 1272-1280.

38. Zubarev, R. A.; Horn, D. M.; Fridriksson, E. K.; Kelleher, N. L.; Kruger N. A.; Lewis, M. A.; Carpenter, B. K.; McLafferty, F. W. Electron Capture Dissociation for Structural Characterization of Multiply Charged Protein Cations. Anal. Chem. 2000, 72, 563-573.

39. Senko, M. W.; Speir, J. P.; McLafferty, F. W. Collisional Activation of Large Multiply-Charged Ions Using Fourier-Transform Mass-Spectrometry. Anal. Chem. 1994, 66, 2801-2808.

40. Little, D. P.; Speir, J. P.; Senko, M. W.; Oconnor, P. B.; McLafferty, F. W Infrared Multiphoton Dissociation of Large Multiply-Charged Ions for Biomolecule Sequencing. Anal. Chem. 1994, 66, 2809-2815.

41. Cooper, H. J.; Hakansson, K.; Marshall, A. G. The Role of Electron Capture Dissociation in Biomolecular Analysis. Mass Spectrom. Rev. 2005, 24, 201-222.

42. Shi, S. D. H. Hemling, M. E. Carr, S. A.; Horn, D. M.; Lindh, I. McLafferty, F. W. Phosphopeptide/Phosphoprotein Mapping by Electron Capture Dissociation Mass Spectrometry. Anal. Chem. 2001, 73, 19-22.

43. Pesavento, J. J.; Mizzen, C. A.; Kelleher, N. L. Quantitative Analysis of Modified Proteins and Their Positional Isomers by Tandem Mass Spectrometry: Human Histone H4. Anal. Chem. 2006, 78, 4271-4280.

44. Solaro, R. J.; Moir, A. J. G.; Perry, S. V. Phosphorylation of Troponinand Inotropic Effect of Adrenaline in Perfused Rabbit Heart. Nature 1976, 262, 615-617.

45. Jideama, N. M. Noland, T. A. Raynor, R. L.; Blobe, G. C. Fabbro, D. Kazanietz, M. G.; Blumberg, P. M.; Hannun, Y. A.; Kuo, J. F. Phosphorylation Specificities of Protein Kinase C Isozymes for Bovine Cardiac Troponin I and Troponin T and Sites Within These Proteins and Regulation of Myofilament properties. J. Biol. Chem. 1996, 271, 2327723283.

46. Noland, T. A.; Guo, X. D.; Raynor, R. L.; Jideama, N. M.; Averyhartfullard, V.; Solaro, R. J.; Kuo, J. F. Cardiac Troponin-I MutantsPhosphorylation by Protein-Kinase-C and Protein-Kinase-a and Regulation of $\mathrm{Ca}^{2+}$-Stimulated MgATPase of Reconstituted Actomyosin S-1. J. Biol. Chem. 1995, 270, 25445-25454.

47. Sumandea, M. P.; Burkart, E. M.; Kobayashi, T.; De Tombe, P. P.; Solaro R. J. Molecular and Integrated Biology of Thin Filament Protein Phosphorylation in Heart Muscle. Ann. N.Y. Acad. Sci. 2004, 1015, 39-52.

48. Wang, H.; Grant, J. E.; Doede, C. M.; Sadayappan, S.; Robbins, J. Walker, J. W. PKC- $\beta$ II Sensitizes Cardiac Myofilaments to $\mathrm{Ca}^{2+}$ by Phosphorylating Troponin I on Threonine-144. J. Mol. Cell. Cardiol. 2006, 41, 823-833.

49. Walker, J. W.; Protein Kinase, C. Troponin I and Heart Failure: Overexpressed, Hyperphosphorylated, and Underappreciated? J. Mol. Cell. Cardiol. 2006, 40, 446-450.

50. Vlahos, C. J.; McDowell, S. A.; Clerk, A. Kinases as Therapeutic Targets for Heart Failure. Nat. Rev. Drug Discov. 2003, 2, 99-113.

51. Whitelegge, J. P.; Zabrouskov, V.; Halgand, F.; Souda, P.; Bassiliana, S.; Yan, W.; Wolinsky, L.; Loo, J. A.; Wong, D. T. W.; Faull, K. F. Protein-Sequence Polymorphisms and Post-Translational Modifications in Proteins from Human Saliva Using Top-Down Fourier-Transform Ion Cyclotron Resonance Mass Spectrometry. Int. J. Mass Spectrom. 2007, 268, 190-197. 